\title{
Les (bonnes) mutations en médecine et en politique
}

\section{Jean Martin}

Dr méd., membre de la rédaction

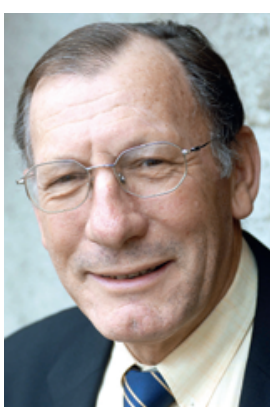

Références

1 Article de M. Eltchaninoff. Journal Le Point, Paris, 27 avril 2017, p. $46-48$

2 "Le simple patient a sa place dans la réflexion éthique». Médecins (Bulletin de l'Ordre national des médecins, Paris), mars-avril 2017, no 48, p. 32

3 «Patients-experts, vers un nouveau métier?» Médecins, no 48, p. 8-9

1 Le terme de patients expert apparaît aussi, de plus en plus, en Suisse dans les média, et de telles personnes sont engagées dans la pratique (ainsi, dans le canton de Vaud dans le suivi du diabète voir www.diabetevaud.ch/ patient-expert).

jean.martin[at]saez.ch
Le nouveau président français a été influencé par les idées de l'éminent philosophe Paul Ricœur dont il a été l'assistant. Dans un article paru au moment de l'élection [1], Michel Eltchaninoff relève que pour Macron, tout aussi éloigné du modèle ultralibéral que du collectivisme uniformisant, l'enjeu n'est pas d'apporter la même chose à tous, c'est de fournir à chacun ce dont il a besoin. Il pense que «la politique consiste à donner aux personnes la capacité de développer leurs potentialités» et préfère, s'agissant d'une ligne de conduite, l'équité à l'égalité à tout crin.

"Je ne crois pas», dit-il, "que la politique doive promettre le bonheur, car elle ne peut tout régir ou tout améliorer. Elle doit plutôt permettre à chacun de trouver sa voie, de devenir le maitre de son destin.» Considérant ce qui précède en pensant aux buts de la médecine et de son exercice, je trouve frappantes les analogies. Mettez médecine à la place de politique et cela marche (cela «colle») très bien. Le médecin ne peut tout régir, il est là pour permettre aux patients qui le consultent de développer au mieux leurs potentialités (cas échéant celles qui leur restent), d'être mieux maître de leurs choix (même si ces derniers sont limités). Et il est essentiel de rechercher ce qui convient à chaque consultant dans ses particularités propres.

Dans le dernier bulletin de l'Ordre national français des médecins, on trouve un texte du Prof. Jean-François Delfraissy [2], nouveau président du Comité consultatif national d'éthique (CCNE). Il a vécu de près l'épopée du sida depuis son émergence au début des années 1980: «Nous avons été une génération de jeunes médecins faisant face à quelque chose de radicalement nouveau: il n'y avait plus d'un côté ceux qui savaient et de l'autre le malade. Il y avait des patients acteurs de leur santé et soutenus par des associations [non-médicales]. Du jamais vu.» Comme médecin cantonal, j’ai vécu intensément cette période. De ce phénomène de désécurisation d'une médecine d'abord impuissante a découlé une évolution comparable à ce que le président français, influencé par Ricœur qui a beaucoup travaillé sur l'éthique sociale, veut aujourd'hui faire en politique. Delfraissy à nouveau: «La dimension technique de notre métier est essentielle mais il reste incomplet si nous ne sommes pas capables d'écouter les personnes et plus largement la société civile. C'est ce que je souhaite proposer au CCNE, certes composé d'experts remarquables, mais le bon sens, et donc le simple citoyen, a sa place dans la réflexion éthique.»

Macron ne veut pas voir le citoyen comme un administré mais comme un acteur à part entière [1]. On a actuellement dans la pratique médicale et des soins un même mouvement, mettant l'accent sur le partenariat entre soignés et soignants et sur la décision partagée. Autre développement décrit dans le Bulletin susmentionné: en France, des Universités des patients (UDP) [3] se sont mises à former des patients-experts ${ }^{1}$ : des malades chroniques qui, forts de leur expérience (que l'université ambitionne de transformer en expertise), mettent ce vécu au profit d'autres malades (s'agissant de diabète, sclérose en plaques, insuffisance rénale, cancers...). Celle de Paris a été la première, en 2010, puis sont venues Marseille et Grenoble. La formation ouvre aux patients-experts des possibilités de se réinsérer socialement et professionnellement. A Paris, 130 d'entre eux ont déjà été diplômés. Toutefois, si un certain nombre ont trouvé en emploi, notamment dans des programmes d'éducation thérapeutique, «le chemin est encore long. A l'hôpital le savant médecin tend à s'opposer au patient profane.»

Je n'ai pas connaissance d'initiatives formalisées de ce genre en Suisse, mais l'article note: «Le concept pourtant n'est pas nouveau. Au Canda, aux USA ou en Suisse, les patients sont sollicités depuis longtemps.» Il est vrai que, chez nous, les diverses formes d'associations de patients sont actives de longue date et ont montré leur potentiel de contributions autonomes à la prise en charge - et à la défense des intérêts - des malades chroniques. Un conseil de Macron en conclusion: «Nous sommes comme recroquevillés sur nos passions tristes, la jalousie, la défiance, la désunion [qu'il voudrait remplacer par] les grandes passions joyeuses, par la liberté, le savoir, l’universel.» On lui souhaite de réussir. 